\title{
Asentamiento larval de la concha de abanico (Argopecten purpuratus) en colectores artificiales en la Bahía Independencia, Pisco, Perú
}

\author{
Ricardo Bandin L. ${ }^{1}$ y Jaime Mendo A. ${ }^{2}$ \\ ${ }^{1}$ Facultad de Ciencias y ${ }^{2}$ Facultad de Pesquería \\ Universidad Nacional Agraria La Molina \\ Apartado 456, Lima 100, Perú \\ E-mail: jmendo@lamolina.edu.pe
}

Recibido 14 noviembre 1996; versión corregida 6 julio 1998; aceptado 8 marzo 1999.

RESUMEN. El presente estudio se efectuó en el área de El Queso, Bahía Independencia (Pisco, Perú), de febrero de 1992 a marzo de 1993. Se usaron colectores de netlón para determinar la distribución vertical del asentamiento larval y se hizo un seguimiento de la captación de semillas en colectores comerciales para determinar el patrón temporal de asentamiento larval de concha de abanico (Argopecten purpuratus). Con el fin de relacionar este patrón con las condiciones ambientales, se registró la temperatura, oxígeno disuelto y transparencia del agua. La distribución vertical de larvas e individuos asentados en los colectores de prueba indicó que la intensidad del asentamiento larval aumenta hasta 45 veces entre los niveles superficial y de fondo, concentrándose entre 11 y $16 \mathrm{~m}$ en una columna de agua de 20 $\mathrm{m}$ de profundidad. La ocurrencia de un período de desove intenso en otoño, fue deducida por máximos de captación en colectores comerciales entre mayo y julio. Se obtuvieron cosechas en colectores comerciales superiores a 500 semillas/bolsa en abril, mayo, junio, julio y octubre en 3 long-lines, lo cual indica importantes períodos de asentamiento ocurridos en la segunda mitad del verano, al inicio y mediados de otoño y a fines de invierno de 1992. Se observó que la captación varía en forma directa al tiempo de exposición de los colectores y se discute la relación entre la variación de los parámetros ambientales y la captación.

Palabras claves: captación de semillas, asentamiento larval, Argopecten purpuratus, Perú, acuicultura.

\section{Larval settlement of the Peruvian scallop (Argopecten purpuratus) on artificial collectors in Independencia Bay, Pisco, Perú}

\begin{abstract}
This study was carried out in El Queso area at the Independencia Bay (Pisco, Perú), from February 1992 to March 1993. The vertical distribution of larvae and temporal pattern of spat settlement of the Peruvian scallop (Argopecten purpuratus) were determined using artificial collectors. In order to relate this pattern with environmental conditions, temperature, dissolved oxigen, and transparency were measured. The distribution of postlarvae and spat settlement pattern in the water column showed up to 45 times more abundance of larvae in the lower than in the upper layer of the water column. Highest abundance of larvae and spat settlement ocurred between 11 and $16 \mathrm{~m}$ depth in a water column of $20 \mathrm{~m}$. Maximum of spat collection in commercial collectors bags were registered in may and july, which suggests a strong spawning activity in autum. Spats collected with commercial bags were higher than 500 spat/ bag in April, May, June, July and October and indicates important periods of spat settlement during the second half of summer season, in autum and during the end of winter 1992. The spat collection varied directly with the exposition time of the bags an is discussed in relation to the environmental parameters.
\end{abstract}

Key words: seed collection, larval settlement, Argopecten purpuratus, Perú, aquaculture. 


\section{INTRODUCCIÓN}

En el Perú la extracción de concha de abanico (Argopecten purpuratus) empieza en la década del 50 (Valdivia y Benites, 1984), mientras que las primeras experiencias en cultivo extensivo se iniciaron en 1979 (Valdivieso, 1990). Ambas actividades se intensificaron con el explosivo incremento poblacional de concha de abanico en todo el litoral, como consecuencia de la ocurrencia del intenso fenómeno El Niño 1982-1983, iniciándose entonces la exportación de este marisco (Benites, 1988, Valdivieso, 1990). Posteriormente la abundancia del recurso se redujo drásticamente por efecto del elevado esfuerzo de pesca y la normalización de las condiciones ambientales (Mendo et al., 1988). Ello condujo a la intensificación de las actividades de cultivo que en un inicio se llevó a cabo transplantando semillas del ambiente natural en concesiones de cultivo de empresas privadas (Benites, 1988)

El cultivo de concha de abanico depende fundamentalmente del abastecimiento de semillas que tiene actualmente dos fuentes principales: la producción en ecloserías y la captación de semillas en el ambiente natural (Benites, 1988; Valdivieso, 1990). Esta última, ampliamente difundida a partir de la experiencia japonesa (Mottet, 1979), se consigue con la instalación de un número apropiado de bolsas colectoras en el lugar y período apropiados; una anticipación equivale a una mayor colonización de fouling, competidores y/o predadores, mientras que un retraso implica perder la mayor parte del grupo larval en asentamiento (Akaboshi y Fuentes 1989; Ventilla, 1982; Quayle, 1981).

Existen varios estudios sobre colección de semillas de A. purpuratus. En la bahía de Tongoy, Pereira et al. (1989) obtuvieron las mayores captaciones en los años 1983, 1985 y 1987 de 230 a 280 semillas/bolsa en períodos de exposición de 3 a 4 meses; mientras que en bahía de Mejillones Avendaño y Cantillanez (1989) utilizando malla mosquitera como sustrato, obtuvieron en verano de 1987 de 113 a 155 semillas por bolsa colectora en períodos de exposición de casi 3 meses. En bahía de Paracas, Vargas (1986) en verano de 1985, utilizó planchas de asbesto cemento como colectores expuestos durante 3 semanas y obtuvo 27 semillas/ plancha; mientras que Robles et al. (1988) con bolsas colectoras expuestas casi 2 meses en verano de 1986, obtuvieron sólo $0,8 \%$ de semillas $A$. purpuratus del total de bivalvos captados. En La
Tunga, bahía Independencia, Ysla y Vargas (1989) usando bolsas colectoras detectaron el máximo de asentamiento en marzo de 1988, obteniendo 1580 semillas por bolsa en un tiempo de exposición de aproximadamente 2 meses. La mayoría de estos estudios han utilizado bolsas colectoras de tipo japonés, usando comúnmente la malla de netlón como sustrato. Sin embargo, los resultados de captación obtenidos en estos estudios son difíciles de comparar debido al uso de diferentes sustratos, tamaños de bolsa y tiempos de exposición; aunque todos ellos coinciden en que la mejor captación se obtiene en la mitad inferior de la columna de agua.

Los objetivos planteados en el presente estudio son evaluar el asentamiento larval a diferentes profundidades de la columna de agua y determinar las variaciones temporales del asentamiento larval.

\section{MATERIALES Y MÉTODOS}

El presente estudio se efectuó en el área denominada El Queso, Bahía Independencia (14¹8'S), que tiene profundidades de 15 a $21 \mathrm{~m}$, ubicada aproximadamente a $48 \mathrm{~km}$ al sur de la ciudad de Pisco (Fig. 1), durante el período de febrero de 1992 a marzo de 1993.

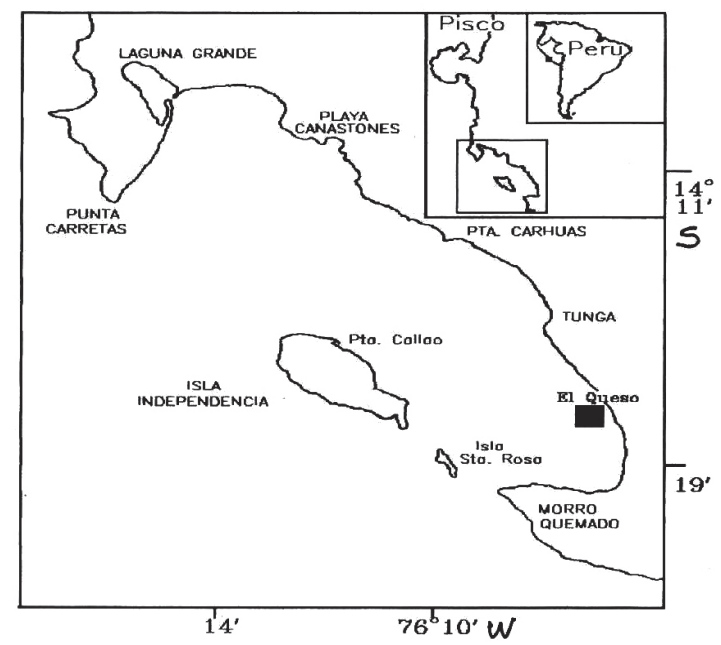

Figura 1. Ubicación del área de estudio.

Figure 1. Location of the study area. 


\section{Distribución vertical de asentamiento larval en bolsas colectoras de prueba}

Entre agosto y diciembre de 1992 se instalaron consecutivamente 8 cabos de $20 \mathrm{~m}$ de longitud, cada uno con 3 colectores de prueba a 3,10 y $17 \mathrm{~m}$ de profundidad. El tiempo de exposición de los colectores varió entre 16 a 41 días; al cabo de los cuales los colectores fueron retirados y reemplazados con otro juego de 3 colectores limpios. Cada colector consistió en una manga de $40 \mathrm{~cm} \mathrm{x} 40 \mathrm{~cm}$ de monofilamento de netlón azul de $16 \mathrm{~mm}$ de abertura de malla con doblez en su parte distal, envuelta por una bolsa de polifilamento de polipropileno verde de $2 \mathrm{~mm}$ con similares dimensiones (Fig. 2). Las postlarvas y semillas fueron desprendidas de la bolsa colectora al agitarla en un recipiente con agua, luego gran parte del fouling se separó por decantación y el material decantado (conteniendo postlarvas y semillas), fue colocado en frascos con formol neutralizado al 2\%. La identificación de los individuos asentados (postlarvas y semillas) se hizo según Akaboshi y Fuentes (1989). Los resultados de las evaluaciones se expresaron en número de individuos asentados por colector.

\section{Asentamiento larval en bolsas colectoras definitivas}

La captación de colectores comerciales se evaluó en el marco de actividades del Centro de Obtención de Semillas de Concha de Abanico (COSCA) del Ministerio de Pesquería, que contaba con 3 sistemas long-line (Fig. 2), denominados L1, L2 y L3, ubicados cada $50 \mathrm{~m}$ en paralelo a la línea de costa, sobre columnas de agua de 15, 18 y $21 \mathrm{~m}$ de profundidad respectivamente. Un long-line posee 100 reinales, cada uno de los cuales se compone de 7 pares de colectores comerciales enlazados por un cabo. Un colector comercial tiene el mismo diseño y materiales que un colector de prueba, pero con el doble de longitud tanto en el sustrato como en la bolsa externa.

La instalación de reinales en un long-line se decidió según la abundancia larval en la columna de agua obtenida mediante arrastres verticales con una red de plancton de $100 \mu$; los reinales quedaron expuestos hasta el inicio de la cosecha, que se determinó de acuerdo a la captación obtenida en colectores piloto. La cosecha de un long-line completo puede tomar de 2 a 5 días, durante los cuales las semi-

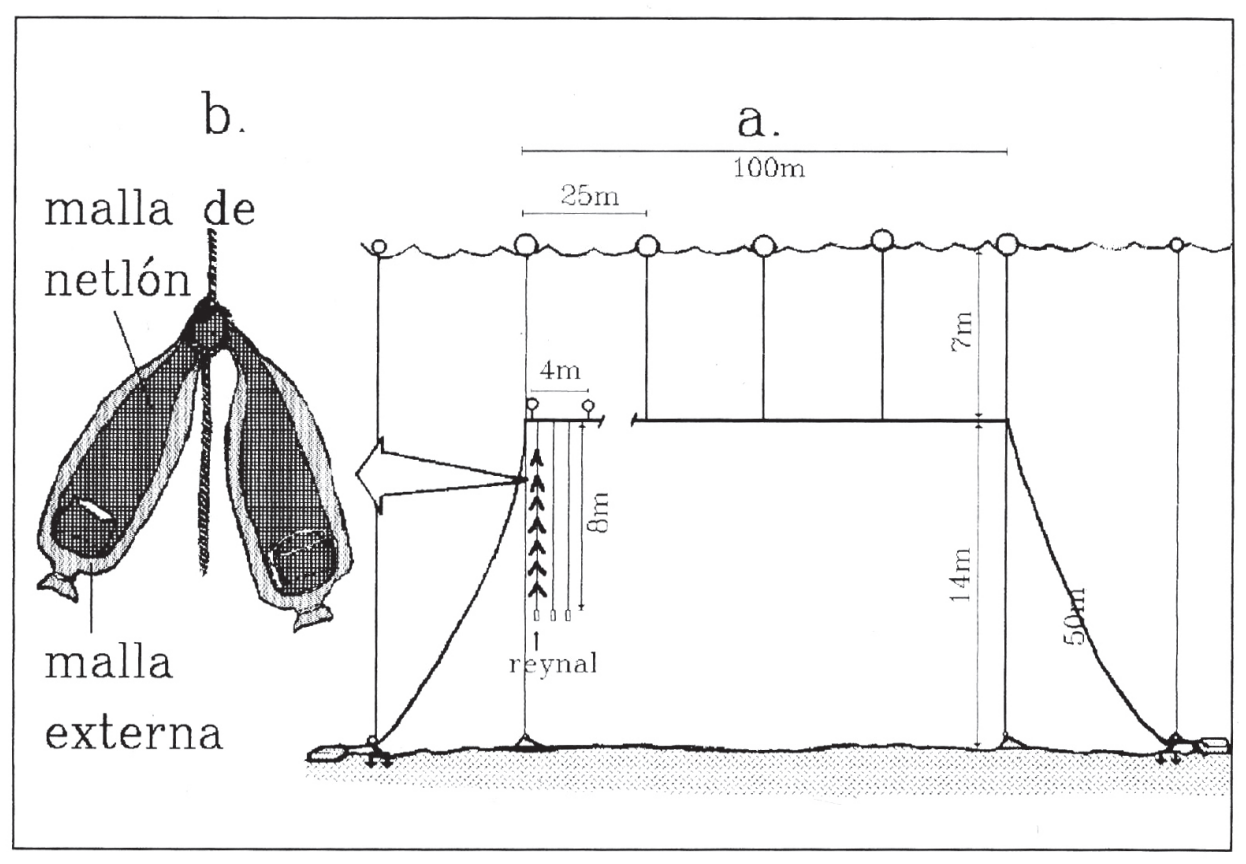

Figura 2. Esquema de un long-line (a) con reinales de bolsas colectoras definitivas (b) para semillas de Argopecten purpuratus.

Figure 2. Scheme of a long-line (a) with commercial collector bags used to collect (b) Argopecten purpuratus seeds. 
llas de A. purpuratus son desprendidas de cada colector comercial y separadas de otros organismos. El conteo se realizó sólo para los individuos mayores de $4 \mathrm{~mm}$. Los individuos menores no fueron considerados en este estudio debido a la dificultad de su cuantificación.

Para establecer los períodos más importantes de asentamiento se definió como buen asentamiento, aquel asociado a una captación igual ó superior a 500 semillas/bolsa (Mottet, 1979). Considerando que la talla de la mayoría de semillas estaba comprendida en el rango de 4 a $8 \mathrm{~mm}$, los períodos de asentamiento importante se dedujeron restando la diferencia entre la edad de las semillas captadas y el período medio de vida larval de 20 días a la fecha de la cosecha (Cisneros et al., 1992).

Adicionalmente, en tres niveles de profundidad de la columna de agua, se midió la temperatura y contenido de oxígeno disuelto, con un oxímetro portátil OXI-96, cuyos valores fueron promediados para toda la columna; además, se estimó la transparencia del agua usando un disco Secchi.

\section{Tratamiento de los datos}

Para evaluar cambios en la distribución vertical de los individuos asentados en los colectores de prueba, se graficaron perfiles de abundancia relativa en superficie, mitad y fondo de la columna de agua. El grado de agregación de los individuos asentados en la columna de agua se estimó con el coeficiente de variabilidad de la media de abundancia $(\mathrm{CV})$, mientras que la profundidad media de ubicación de los mismos se estimó con el centro de masa ZCM (Tremblay y Sinclair, 1990), calculada según:

$$
\mathrm{ZCM}=\Sigma \mathrm{p}_{\mathrm{i}} \mathrm{z}_{\mathrm{i}}
$$

donde $\mathrm{p}_{\mathrm{i}}=$ proporción del número total de organismos presentes en el nivel $i, y z_{i}=$ profundidad en el nivel i.

Asimismo, se graficó el nivel de captación versus el tiempo de exposición, con los resultados tanto de los colectores de prueba como de colectores comerciales, para determinar si existe relación entre estas variables. Además, se aplicaron análisis de correlación lineal entre la captación media de colectores comerciales por campaña y los promedios de temperatura y oxígeno disuelto para la columna de agua. Finalmente, para determinar si existen diferencias significativas entre los promedios del número de semillas colectadas/bolsa/día de exposición entre los 3 long-lines, se aplicó un análisis de varianza al nivel de significancia $\mathrm{a}=0,05$ (Zar, 1984).

\section{RESULTADOS Y DISCUSIÓN}

\section{Algunas características del área de estudio}

El Queso es un área de aguas someras de 15-21 m de profundidad, con fondo de arena, cieno y conchuela. La temperatura media para la columna de agua (TC) registró un patrón de variación con máximos entre los 22 y $23^{\circ} \mathrm{C}$ en febrero y marzo, y mínimos de $15^{\circ} \mathrm{C}$ en julio, agosto y octubre (Fig. 3a). Datos adicionales de promedios mensuales de temperatura superficial de orilla (TS) registrados por Pesca Perú en la isla Independencia muestran una tendencia similar y al compararlos con el patrón promedio mensual propuesto por Morón et al. (1988), evidencian anomalías positivas entre enero y junio de hasta $5,2^{\circ} \mathrm{C}$ en marzo. El oxígeno disuelto promedio para la columna (ODC) varió de 2,0 a $7,8 \mathrm{mg} / \mathrm{l}$, mientras que la transparencia del agua fluctuó de 3 a 9 m, determinándose los mayores valores en verano (Figs. 3b y c). Estos elementos sugieren que en 1992 se manifestó un fenómeno El Niño moderado.

\section{Distribución vertical del asentamiento larval}

La proporción de abundancia en los tres niveles de profundidad, de ejemplares de A. purpuratus asentados en colectores de prueba mostró escasa variación, siendo la abundancia en el fondo hasta 45 veces mayor que la de superficie. La abundancia promedio en la columna de agua mostró sus máximos valores en octubre (1563 individuos/bolsa) y mediados de diciembre (1927 individuos/bolsa). Los valores de ZCM variaron entre 12 y $16 \mathrm{~m}$ de profundidad y los CV más altos (> 100\%) se presentaron especialmente en agosto, octubre y noviembre (Tabla 1, Fig. 4). Resultados similares obtuvieron Ysla y Vargas (1989), quienes muestran perfiles de abundancia obtenidos con colectores de prueba instalados en La Tunga a 10, 14 y $18 \mathrm{~m}$ de profundidad, de lo que se deduce un ZCM de asentamiento de 15,6 a $18 \mathrm{~m}$. Al respecto, Illanes et al. (1985) obtuvieron la mayor abundancia entre los 15 y $16 \mathrm{~m}$ de profundidad en la bahía de Tongoy.

También, en los tres niveles de profundidad se observó la presencia de pedivelígeras y algunas 

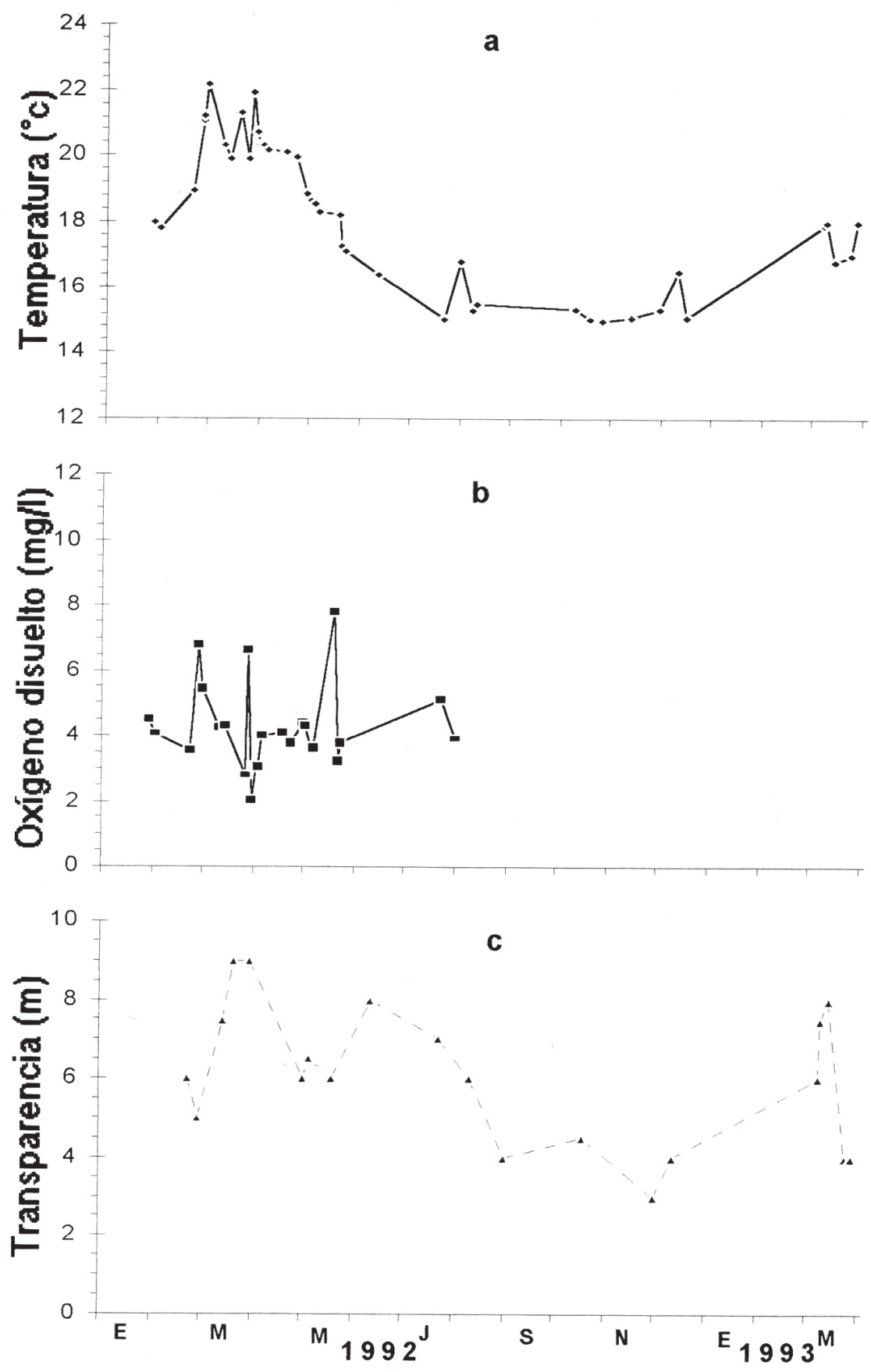

Figura 3. Promedios para la columna de agua de temperatura (a) y oxígeno disuelto (b), y transparencia (c) en El Queso, desde enero de 1992 a marzo de 1993.

Figure 3. Water column average of temperature (a), dissolved oxygen (b) and transparency (c) in EI Queso, from january 1992 to march 1993. 
Investigaciones Marinas, Vol. 27 - 1999
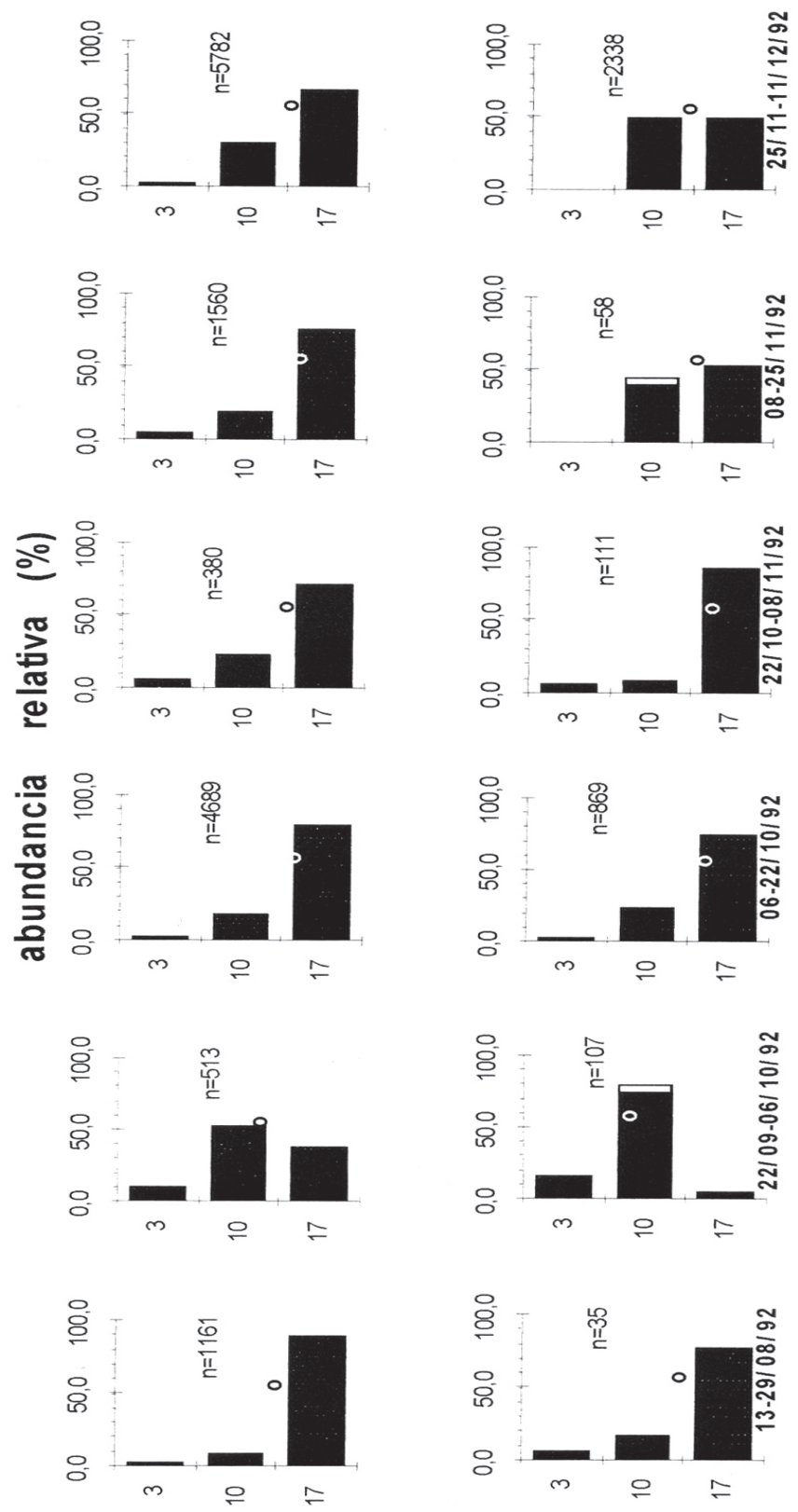

\section{(w) pep!punjoid}

Figura 4. Distribución vertical de abundancia de individuos asentados (a) y larvas (b) de A. purpuratus en colectores de prueba instalados en El Queso durante diferentes períodos de exposición, de agosto a noviembre de 1992. En (b) las barras oscuras representan pedivelígeras y las claras veliconchas; "n" indica el total de individuos para los 3 niveles.

Figure 4. Verticla distribution of sttled individuals (a) and larvae (b) abundance of Argopecten purpuratus in experimental collectors installed in El Queso in different period, from august to november of 1992. Dark bars indicate pediveligers and white bars indicate veliconch; " $n$ " indicates total of individuals for the 3 levels. 
veliconchas en los colectores de prueba cuya distribución vertical y patrón de variación de abundancia fueron similares a los de los individuos asentados. En el análisis de las muestras se registró, además de individuos asentados, una cantidad apreciable de larvas de A. purpuratus cuyo ZCM fue similar al de los asentados (Tablas 1 y 3), lo que concuerda con
Brand et al. (1980), que sugieren que la abundancia de individuos asentados en los colectores es reflejo del comportamiento larval. Sin embargo, se debe mencionar que a $3 \mathrm{~m}$, la captación puede haber sido afectada por el fouling que disminuye el área de sustrato para la fijación.

Tabla 1. Captación (asentados/bolsa) en tres profundidades con colectores de prueba instalados en EI Queso (agosto 1992 - enero 1993). A= número de asentados/bolsa; $\tilde{n}=$ promedio de las tres profundidades; $P v$ y Vc= pedivelígeras y veliconchas encontradas en los colectores; $\mathrm{CV}=$ coeficiente de variabilidad y $\mathrm{ZCM}=$ centro de masa (- = no hay datos).

Table 1. Number of scallops per bag collected at three depths using experimental collectors installed in EI Queso (August 1992 - January 1993). $A=$ number; $\tilde{n}=$ average for the three depths; $P_{V}$ and $V_{c}=$ number of scallops in pediveliger and veliconch stages; $\mathrm{CV}=$ variation coefficient and $\mathrm{ZCM}=$ mass center (- = no data).

\begin{tabular}{|c|c|c|c|c|c|c|c|c|c|c|c|c|c|c|c|c|}
\hline \multirow{3}{*}{$\begin{array}{l}\text { Fecha } \\
\text { de } \\
\text { Instal. }\end{array}$} & \multirow{3}{*}{$\begin{array}{c}\text { días } \\
\text { de } \\
\text { exp. }\end{array}$} & \multicolumn{9}{|c|}{ Profundidad (m) } & \multicolumn{3}{|c|}{$\mathrm{Pv}+\mathrm{Vc}$} & \multicolumn{3}{|c|}{ Asentados } \\
\hline & & \multicolumn{3}{|c|}{3} & \multicolumn{3}{|c|}{10} & \multicolumn{3}{|c|}{17} & \multirow[t]{2}{*}{$\tilde{\mathrm{n}}$} & \multirow{2}{*}{$\begin{array}{l}\mathrm{CV} \\
(\%)\end{array}$} & \multirow{2}{*}{$\begin{array}{c}\mathrm{ZCM} \\
(\mathrm{m})\end{array}$} & \multirow[t]{2}{*}{$\tilde{\mathrm{n}}$} & \multirow{2}{*}{$\begin{array}{l}\mathrm{CV} \\
(\%)\end{array}$} & \multirow{2}{*}{$\begin{array}{r}\mathrm{ZCM} \\
(\mathrm{m})\end{array}$} \\
\hline & & $\mathrm{PV}$ & $\mathrm{Vc}$ & A & $\mathrm{PV}$ & $\mathrm{Vc}$ & A & $\mathrm{PV}$ & $\mathrm{Vc}$ & A & & & & & & \\
\hline $13 / 08 / 92$ & 16 & 2 & 0 & 22 & 6 & 0 & 103 & 27 & 0 & 1036 & 11,7 & 115,1 & 15,0 & 387,0 & 145,6 & 16,1 \\
\hline $29 / 08 / 92$ & 24 & 1 & 9 & 15 & - & - & - & 36 & 153 & 1136 & - & - & - & - & - & - \\
\hline $22 / 09 / 92$ & 14 & 17 & 0 & 50 & 79 & 6 & 271 & 5 & 0 & 192 & 35,7 & 121,0 & 9,2 & 171,0 & 65,5 & 11,9 \\
\hline $06 / 10 / 92$ & 16 & 22 & 0 & 133 & 203 & 0 & 835 & 644 & 0 & 3721 & 289,7 & 110,4 & 15,0 & 1563,0 & 121,7 & 15,4 \\
\hline $22 / 10 / 92$ & 20 & 6 & 1 & 24 & 9 & 0 & 88 & 95 & 0 & 268 & 37,0 & 135,8 & 15,5 & 126,7 & 99,9 & 14,5 \\
\hline $08 / 11 / 92$ & 17 & 1 & 0 & 80 & 23 & 3 & 305 & 31 & 0 & 1175 & 19,3 & 83,1 & 13,6 & 520,0 & 111,2 & 14,9 \\
\hline $25 / 11 / 92$ & 17 & 7 & 0 & 174 & 1158 & 8 & 1742 & 1160 & 5 & 3866 & 779,3 & 85,8 & 13,5 & 1927,3 & 96,1 & 14,5 \\
\hline $11 / 12 / 92$ & 41 & - & - & - & - & - & - & 12 & 0 & 2970 & - & - & - & - & - & - \\
\hline
\end{tabular}

Tabla 2. Captación en bolsas colectoras definitivas en El Queso (febrero 1992 - enero 1993). Tipos de malla externa, $\mathrm{j}=$ japonesa $\mathbf{y} \mathrm{ch}=$ chilena.

Table 2. Spats collected using commercial collector bags at El Queso (February 1992 - January 1993); j and ch indicate the use of collectors with japanese and chilean external net respectively.

\begin{tabular}{|c|c|c|c|c|c|c|c|c|}
\hline \multicolumn{2}{|c|}{ Fecha } & \multirow{2}{*}{$\begin{array}{c}\text { Exposición } \\
\text { promedio } \\
\text { (días) }\end{array}$} & \multirow{2}{*}{$\begin{array}{l}\text { Long- } \\
\text { line }\end{array}$} & \multirow{2}{*}{$\begin{array}{c}\mathbf{N}^{\circ} \\
\text { reinales }\end{array}$} & \multicolumn{3}{|c|}{$\mathrm{N}^{\circ}$ de semillas (sem) mayores a $4 \mathrm{~mm}$} & \multirow{2}{*}{$\begin{array}{c}\text { Malla } \\
\text { externa }\end{array}$} \\
\hline Instalación & Recojo & & & & Total & sem/bolsa & $\begin{array}{l}\text { sem/ bolsa/ } \\
\text { día }\end{array}$ & \\
\hline $07 / 02$ & $25-28 / 03$ & 48,5 & L3 & 100 & 147.503 & 105,4 & 2,17 & $\mathrm{j}$ \\
\hline $16 / 02$ & $01-03 / 04$ & 46,0 & L2 & 66 & 319.998 & 346,3 & 7,53 & $\mathrm{j}$ \\
\hline $12 / 03$ & $21-24 / 04$ & 41,5 & L1 & 79 & 297.454 & 268,9 & 6,48 & $\mathrm{j}$ \\
\hline $29 / 03$ & $12-16 / 05$ & 46,0 & L3 & 106 & 503.275 & 339,1 & 7,37 & $\mathrm{j}$ \\
\hline $10 / 04$ & $20 / 05-05 / 06$ & 44,4 & L2 & 91 & 293.976 & 230,8 & 5,20 & $\mathrm{j} / \mathrm{ch}$ \\
\hline $29 / 04$ & 24/06-02/07 & 60,3 & L1 & 88 & 816.995 & 663,1 & 11,01 & $\mathrm{j} / \mathrm{ch}$ \\
\hline $20 / 05$ & 04-07/08 & 76,5 & L3 & 86 & 85.032 & 70,6 & 0,92 & $\mathrm{Ch}$ \\
\hline $12 / 06$ & 01-09/09 & 85,4 & L2 & 93 & 424.953 & 326,4 & 3,82 & $\mathrm{ch}$ \\
\hline $31 / 07$ & $20-22 / 10$ & 82,0 & L1 & 76 & 293.989 & 276,3 & 3,37 & $\mathrm{ch}$ \\
\hline $20 / 08$ & $03-05 / 11$ & 76,0 & L3 & 80 & 110.586 & 98,7 & 1,30 & $\mathrm{ch}$ \\
\hline $23 / 09$ & $08-11 / 12$ & 77,5 & L2 & 82 & 129.494 & 112,8 & 1,46 & $\mathrm{ch}$ \\
\hline $23 / 10$ & $12-13 / 01 / 93$ & 81,5 & L1 & 82 & 97.993 & 85,4 & 1,05 & $\mathrm{ch}$ \\
\hline
\end{tabular}




\section{Variación temporal del asentamiento larval}

La abundancia de semillas por colector por día de cosecha, varió de 64 a 1.428 semillas/bolsa, registrándose las mejores cosechas (>500 semillas/bolsa) en otoño en L2 (3 de abril, 5 de junio) y L3 (15 y 16 de mayo) y en invierno ( 1 y 2 de julio) y primavera (22 de octubre) en L1. Las cosechas más bajas (<100 semillas/bolsa) se registraron en verano en L1 (12 y 13 de enero de 1993), en otoño (25 y 26 de marzo) e invierno (4 al 7 agosto) en L3 y en primavera en L3 (3 de noviembre) y L2 (10 de diciembre) (Fig. 5). En base a estos resultados y a la edad de las semillas, se estimaron dos períodos importantes de asentamiento larval: a) todo el verano (especialmente febrero y marzo) y primera mitad del otoño, y b) fines de otoño y primera mitad del invierno. Estos períodos tienen similitud con los períodos de asentamiento larval de A. purpuratus de la bahía de Paracas estimados por Wolff (1988) mediante el análisis de frecuencias de longitudes, quien propone que el éxito de una clase anual depende más de las condiciones que favorecen el desarrollo y asentamiento larval, que de aquellas que determinan la maduración y fecundidad de los bancos parentales. El estudio realizado por Ysla y Vargas (1989), muestra una alta concordancia con el período de asentamiento observado en verano en el presente estudio.

La mejor cosecha promedio por campaña (>500 semillas/bolsa) ocurrió a inicios de invierno en L1 (24 de junio/2 de julio) siendo el resultado del asentamiento de otoño, mientras que las cosechas más bajas ocurrieron a mediados de invierno (4/7 de agosto) y a mediados de primavera (3/5 de noviembre) en L3 y en verano (12/13 de enero de 1993) en L1 (Tabla 2). El máximo de 1.428 semillas/bolsa (> de $4 \mathrm{~mm}$ ) sugiere que la cosecha total debió ser superior al total de 1.580 asentados/bolsa obtenido por Ysla y Vargas (1989) en La Tunga, y muy superior al máximo de 155 asentados/bolsa obtenido por Avendaño y Cantillanez (1989) en bahía de Mejillones. El promedio general de la cosecha anual para el período evaluado, considerando un tiempo medio de exposición de dos meses, fue de 267 semillas/bolsa, que corresponde a una captación total muy superior a los máximos obtenidos en 1982 y

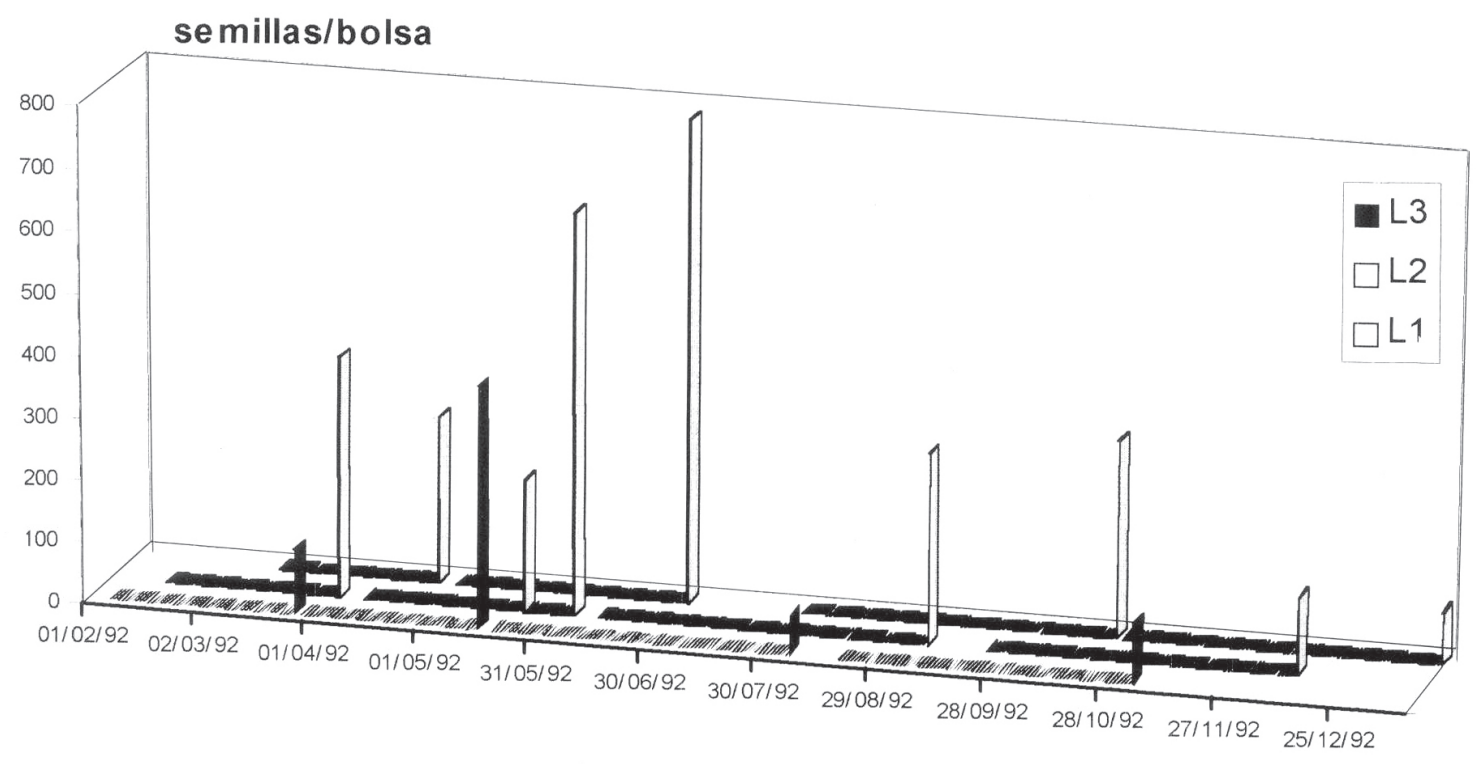

Figura 5. Número de semillas ( $>4 \mathrm{~mm})$ de $A$. purpuratus, captadas en los 3 long-lines con colectores comerciales. El Queso, febrero de 1992 - Enero de 1993. El extremo derecho de cada "sombra" indica la fecha de instalación.

Figure 5. Number of Argopecten purpuratus seeds ( $>4 \mathrm{~mm}$ ), collected in the 3 long-lines with artificial collectors. El Queso, february 1992 - january 1993. The right end of each "shadow" indicates the exposure time of collectors. 
1987 por Pereira et al. (1989), de 230 a 280 individuos/bolsa en períodos de exposición de 3-4 meses en bahía de Tongoy. Todo ello indica el gran potencial que tiene el área de El Queso para fines de captación comercial a gran escala.

Aun cuando el fenómeno El Niño parece favorecer la cosecha de semillas de concha de abanico (Illanes et al., 1985), también se han obtenido buenos rendimientos en años fríos (Pereira et al., 1989). Probablemente, los patrones temporales de asentamiento y reclutamiento naturales pueden ser mejor explicados en función de cambios en la circulación de agua y alteraciones en los vientos, que ya han sido señalados como factores responsables del éxito de asentamiento larval y reclutamiento de la concha de abanico (Mendo et al., 1989) y otros invertebrados bentónicos (Roughgarden et al., 1988; Tremblay y Sinclair, 1991).

Los análisis de correlación lineal simple entre la abundancia de semillas/bolsa en función de la TC y del ODC no resultaron significativos, mientras que en función del tiempo de exposición resultan altamente significativos al separar los datos en dos grupos, el primero con tiempos de exposición inferiores a 65 días $(\mathrm{r}=0,70 ; \mathrm{P}<0,01)$ y el segundo con tiempos de exposición superiores a 65 días $(\mathrm{r}=0,78$; $\mathrm{P}<0,01$ ) (Tabla 3, Fig. 6). Esto podría indicar que el asentamiento larval es permanente y genera una acumulación de asentados hasta los 65 días de exposi-
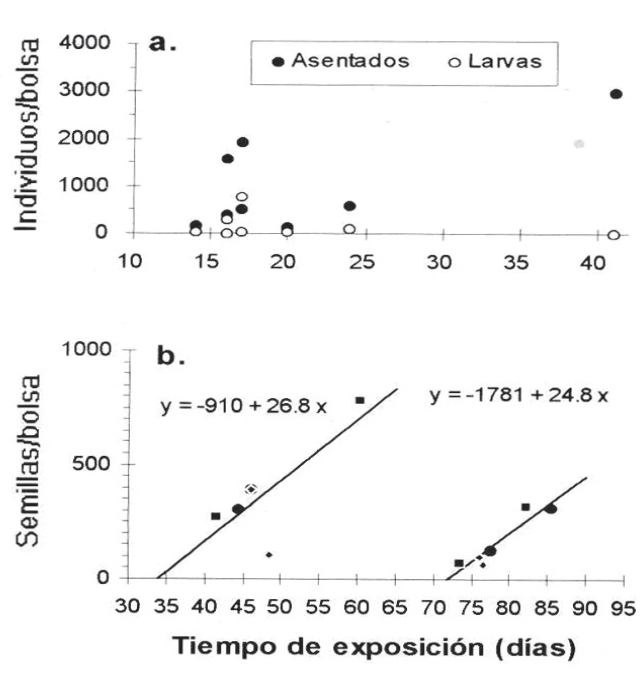

Figura 6. Relación entre captación y tiempo de exposición en colectores de prueba (a.) y en colectores definitivos (b.) para Argopecten purpuratus. En (a) número de post-larvas y larvas en los niveles superficial, medio y fondo respectivamente. En (b) L1, L2 y L3 representan los long-lines tratados.

Figure 6. Relationships between seed collection and exposure time in experimental (a) and in commercial (b) collectors for Argopecten purpuratus. (a.) number of post-larvae at surface, medium abd bottom levels respectively. (b.) $\mathrm{L} 1, \mathrm{~L} 2$ and $\mathrm{L} 3$ indicate the different long-lines.

Tabla 3. Resultados de los análisis de correlación realizados. n.s., * y ** indican: no significativo $(\mathbf{P}>\mathbf{0 , 0 5})$, significativo $(0,01<\mathrm{P}<0,05)$ y altamente significativo $(\mathrm{P}<0,01)$ respectivamente.

Table 3. Results of the simple linear correlation analysis carried out. ns, * and ** means: non significants $(\mathbf{P}>$ $0,05)$, significant $(0,01<\mathrm{P}<0,05)$ y highly significant $(\mathrm{P}<0,01)$ respectively.

Colectores de Prueba
\begin{tabular}{|lcccc|}
\hline ñ asentados/bolsa vs. TC & r & P & Significancia \\
ñ asentados/bolsa vs. tiempo de exposición & 6 & 0,62 & $0,1<\mathrm{P}<0,2$ & n.s. \\
ñ asentados/bolsa vs ñ larvas/bolsa & 6 & $-0,07$ & $\mathrm{P}>0,5$ & n.s. \\
ZCM asentados vs ZCM larval & 6 & 0,91 & $0,01<\mathrm{P}<0,02$ & $*$ \\
\hline Colectores comerciales & 6 & 0,89 & $0,01<\mathrm{P}<0,02$ & $*$ \\
\hline Prom. semillas/bolsa vs. TC & $\mathbf{n}$ & $\mathbf{r}$ & $\mathbf{P}$ & Significancia \\
Prom. semillas/bolsa vs TC, (t expo.<65días) & 6 & $-0,79$ & $0,1<\mathrm{P}<0,2$ & n.s. \\
Prom. semillas/bolsa vs TC, (t expo.>65días) & 6 & $-0,21$ & $\mathrm{P}>0,5$ & n.s. \\
Prom. semillas/bolsa vs. ODC & 8 & 0,05 & $\mathrm{P}>0,5$ & n.s. \\
Prom. semillas/bolsa vs ODC, (t expo.<65días) & 6 & $-0,14$ & $\mathrm{P}>0,5$ & n.s. \\
semillas/bolsa vs t. expo. (<65días) & 6 & 0,78 & $\mathrm{P}<0,001$ & $* *$ \\
semillas/bolsa vs t. expo. ( $>65$ días) & 6 & 0,93 & $\mathrm{P}<0,001$ & $* *$ \\
\hline
\end{tabular}


Tabla 4. Análisis de varianza de la captación (semillas/bolsa/día) por long-line de colectores comerciales (ver la $8^{\text {va }}$ columna de la Tabla 2 ).

Table 4. Variance analysis of the spat collection (seed/bag/day) in different long-lines using commercial collectors (see the $8^{\text {th }}$ column of the Table 2).

\begin{tabular}{|cccccc|}
\hline Fuentes de variación & SC & GL & CM & F cal & P \\
\hline Total & 115,10 & $\mathrm{~N}-1=11$ & & 0,5765 & 0,5805 \\
Grupos & 13,07 & $\mathrm{k}-1=2$ & 6,54 & & \\
Error & 102,03 & $\mathrm{~N}-\mathrm{k}=9$ & 11,34 & & \\
\hline
\end{tabular}

$\mathrm{SC}=$ suma de cuadrados; $\mathrm{GL}=$ grados de libertad; $\mathrm{CM}=$ cuadrado medio

ción (primer grupo), después de los cuales se manifestaría un efecto de mortalidad denso-dependiente que deja el área disponible para nuevos asentamientos (segundo grupo).

Con el ANOVA (Tabla 4), aunque no se encontraron diferencias significativas entre los promedios de captación de cada long-line, no puede desestimarse que la posición de los reinales en la columna de agua tenga influencia sobre la captación, ya que de acuerdo a la prueba de poder del ANOVA, el número de repeticiones usadas (campañas) debió ser mucho mayor $(n=37)$ para la prueba de hipótesis planteada, lo que hubiese demandado un seguimiento de 4 años. Al respecto, Avendaño y Cantillanez (1992) encontraron diferencias significativas en las cosechas de colectores instalados en los $7 \mathrm{~m}$ cercanos al fondo en columnas de agua de 17,22 y $27 \mathrm{~m}$, observando un mayor rendimiento a $\operatorname{los} 22 \mathrm{~m}$.

\section{AGRADECIMIENTOS}

Al personal del Centro de Obtención de Semillas de Concha de Abanico (COSCA) por su apoyo al brindar los medios e infraestructura necesaria para el trabajo de campo y facilitar los datos de producción. A los biólogos Oscar Carranza, Héctor Fiestas y al Ing. Luis Ysla por el adiestramiento en las técnicas de laboratorio y su apoyo durante las evaluaciones. A los investigadores de los departamentos de Acuicultura de las Universidades Católica del Norte y de Antofagasta (Chile), quienes realizaron lecturas críticas de este trabajo, y a todas aquellas personas que de una u otra forma contribuyeron con su ejecución.

\section{REFERENCIAS}

Akaboshi, S. e I. Fuentes. 1989. Determinación del momento de asentamiento larval. $2^{\circ}$ Curso Internacional de Cultivo de Moluscos. Coquimbo, Chile, $16 \mathrm{pp}$.

Avendaño, M. y M. Cantillanez. 1989. Observaciones sobre captación de semilla de concha de Argopecten purpuratus (Lamarck, 1819) en la Bahía de Mejillones del Sur, Chile. Estud. Oceanol., 8: 51-59.

Avendaño, M. y M. Cantillanez. 1992. Colecta artificial de semilla de Argopecten purpuratus (Lamarck, 1819) en Bahía de Mejillones, Chile. II. Observaciones sobre profundidad óptima de captación. Estud. Oceanol., 11: 39-43.

Benites, C. 1988. El desarrollo de la maricultura en el Perú con énfasis en la concha de abanico (Argopecten purpuratus) y langostinos (Pennaeus vannamei), pp. 196-201. In: H. Salzwedel y Landa (eds.). Recursos y dinámica del ecosistema de afloramiento peruano. Bol. Inst. Mar Perú-Callao, Vol. Extr., 382 pp.

Brand, A.R. 1991. Scallop ecology: Distributions and behaviour. In: S. Shumway (ed.). Scallops: biology, ecology and aquaculture. Developments in Aquaculture and Fisheries Science, 21: 517-584.

Brand, A.R., J. Paul y J. Hoogester. 1980. Spat settlement of the scallops Chlamys opercularis (L.) and Pecten maximus (1.) on artificial collectors. J. Mar. Biol. Ass. U.K., 60: 379-390.

Cisneros, R., V. Valdivieso y J. Bautista. 1992. Desarrollo larvario de Argopecten purpuratus L. procedentes de reproductores parasitados. Memoria X CONABIOL 2-7 agosto 1992, Lima, Perú: 345349. 
Illanes, J.E., S. Akaboshi y E. Uribe. 1985. Efectos de la temperatura en la reproducción del ostión del norte Chlamys (Argopecten) purpuratus en la Bahía Tongoy durante el fenómeno El Niño 1982-83. Invest. Pesq. (Chile), 32: 167-173.

Mendo, J., V. Valdivieso y C. Yamashiro. 1988. Cambios en densidad, número y biomasa de la población de concha de abanico (Argopecten purpuratus) en la Bahía Independencia (Pisco, Perú) durante 1984-87. pp. 153-162. In: H. Salzwedel y Landa (eds.). Recursos y Dinámica del ecosistema de afloramiento peruano. Bol. Inst. Mar Perú-Callao, Vol. Extr., 382 pp.

Mendo, J., C. Yamashiro, J. Rubio, A. Kameya, E. Jurado, M. Maldonado y S. Guzmán. 1989. Evaluación de la población de concha de abanico (Argopecten purpuratus) en B. Independencia, Pisco, Perú del 23 de setiembre al 9 de octubre de 1987. Informe 94, Inst. Mar Perú-Callao, 65 pp.

Morón, O., N. Lostanau y L. Escudero. 1988. Parámetros oceanográficos en Bahía Independencia, entre mayo de 1985 y julio de 1987. pp. 153162. In: H. Salzwedel y Landa (eds.). Recursos y Dinámica del ecosistema de afloramiento peruano. Bol. Inst. Mar Perú-Callao, Vol. Extr., 382 pp.

Mottet, M.G. 1979. A review of the fishery biology and culture of scallops. Tech. Rep. 39, Department of Fisheries, Washington, USA, 100 pp.

Navarro, R., L. Sturia, O. Cordero y M. Avendaño. 1991. Aquaculture and fisheries in Chile. In: $\mathrm{S}$. Shumway (ed.). Scallops: Biology, Ecology and Aquaculture. Developments in Aquaculture and Fisheries Science, 21: 1001-1016.

Pereira, L. 1989. Cultivo del ostión del norte (Argopecten purpuratus) en ambiente natural. Man, $2^{\circ}$ Curso Internacional de Cultivo de Moluscos. Coquimbo, Chile, 36 pp.

Pereira, L., J.E. Illanes y S. Akaboshi. 1989. Captación de semilla en ambiente natural del ostión del norte Argopecten purpuratus en Bahía Tongoy IV Región, Chile. Anales Científicos UNALM (Lima, Perú), Vol Extr. Memorias del $2^{\circ}$ COLACMAR, 17-21 agosto 1987: 281-292.
Quayle, D.B. 1981. Tropical oysters: culture and methods. CIID, Ontario, Canadá. 84 pp.

Robles, A., M. Méndez y G. Sánchez. 1988. Captación de moluscos en Bahía Paracas, Perú. pp. 177. 180. In: H. Salzwedel y Landa (eds.). Recursos y Dinámica del ecosistema de afloramiento peruano. Bol. Inst. Mar Perú-Callao, Vol. Extr., 382 pp.

Roughgarden, J., S. Gaines y H. Possingham. 1988. Recruitment dynamics in complex life cycles. Science 241: 1397-1560.

Tremblay, M.J. y M. Sinclair. 1990. Diel vertical migration of sea scallop larvae Placopecten magellanicus in a shallow embayment. Mar. Ecol. Prog. Ser., 67: 19-25.

Tremblay, M.J. y M. Sinclair. 1991. Inshore-offshore differences in the distribution of sea scallop larvae: implications for recruitment (extracto). ICES mar. Sci. Symp., 192: 39 pp.

Valdivia, E. y C. Benites. 1984. Informe sobre la prospección del recurso concha de abanico en la zona de Pisco. Infor. Inter. Inst. Mar Perú-Callao, 13 pp.

Valdivieso, V. 1990. Cultivo de moluscos en el Perú. En: Hernández R. (ed.). Cultivo de Moluscos en América Latina - Memorias de la $2^{\circ}$ Reunión del Grupo de Trabajo Técnico en Ancud-Chiloe-Chile. Ed. Guadalupe. Bogotá-Colombia, pp. 329-343.

Vargas, J.M. 1986. Técnicas de captación de semilla de concha de abanico Argopecten purpuratus en la Bahía de Paracas. Tesis, Ing. Pesq. Univ. Nac. Agraria La Molina, 54 pp.

Ventilla, R.F. 1982. The scallop industry in Japan. Adv. Mar. Biol., 20: 309-382.

Wolff, M. 1988. Spawning and recruitment in the Peruvian scallop Argopecten purpuratus. Mar. Ecol. Prog. Ser., 42: 213-217.

Ysla, L. y J. Vargas. 1989. Informe sobre el proyecto de captación de larvas de concha de abanico (Argopecten purpuratus) en la zona de Pisco. Bol. 28, Fac. Pesq., Univ. Nac. Agraria La Molina, Lima, Perú: 41-67.

Zar, J. 1984. Biostatistical analysis. Prentice Hall Inc., $718 \mathrm{pp}$. 